\title{
NON-REFLEXIVE DOUBLE TRIANGLES
}

\author{
W. E. LONGSTAFF
}

(Received 20 September 1982)

Communicated by R. Vyborny

\begin{abstract}
A double triangle subspace lattice in a Hilbert space $H$ is a 5-element set of subspaces of $H$, containing (0) and $H$, with each pair of non-trivial elements intersecting in (0) and spanning $H$. It is shown that if any pair of non-trivial elements has a closed vector sum the double triangle is both non-reflexive and non-transitive. A double triangle in $H \oplus H$ is an operator double triangle if each non-trivial element is the graph of an operator acting on $H$. A sufficient condition is given for any operator double triangle to be non-reflexive.
\end{abstract}

1980 Mathematics subject classification (Amer. Math. Soc.): primary 47 A 15; secondary 06 A 20.

\section{Introduction}

Throughout this paper $H$ denotes a complex non-zero Hilbert space, 'subspace' means closed linear manifold, 'operator' means bounded linear transformation and every lattice of subspaces of $H$ contains $(0)$ and $H$. For a lattice $e$ of subspaces of $H$, Alg $\mathcal{L}$ denotes the set of operators on $H$ which leave every element of $\mathcal{L}$ invariant and Lat $\mathrm{Alg} \mathcal{L}$ denotes the set of subspaces of $H$ invariant under every element of $A \lg \mathscr{L}$. Of course, $\mathcal{L} \subseteq$ Lat $\mathrm{Alg} \mathcal{L}$. If equality holds $\mathcal{L}$ is

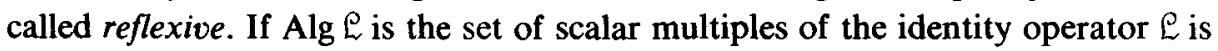
called transitive. This notation and terminology is due to P. R. Halmos [4], [5]. In view of their special algebraic importance [8, page 90] two finite lattice types deserve immediate attention when the questions of reflexivity and transitivity are raised namely the pentagon and the double triangle. Their Hasse diagrams are, 
respectively,

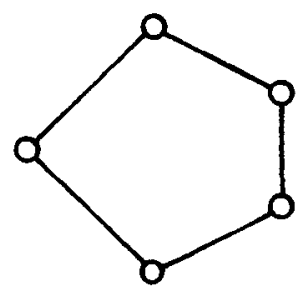

and

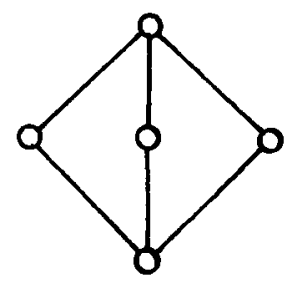

It is shown in [5] that reflexive pentagons and non-reflexive double triangles occur. That non-reflexive pentagons occur is shown in [7]. Concerning transitivity, it follows easily from Proposition 3.1 of [6] that no pentagon is transitive. The questions: Does there exist a reflexive (respectively, a transitive) double triangle? remain open. The present paper discusses, primarily, the former question. For a recent discussion of the latter see [2].

Thus the lattices considered are those of the form $\mathscr{D}=\{(0), K, L, M, H\}$ where the subspaces $K, L, M$ of $H$ satisfy $K \cap L=K \cap M=L \cap M=(0)$ and $K \vee L=K \vee M=L \vee M=H$ (' $\vee$ ' denotes closed linear span). First, a proof is given of the 'folk theorem' that $\mathscr{D}$ is non-reflexive if any of the vector sums $K+L, K+M, L+M$ is closed. The same condition on vector sums is shown to guarantee non-transitivity. The remainder concerns double triangles in $H \oplus H$ of the form $\{(0), G(A), G(B), G(C), H \oplus H\}$ where $A, B, C$ are operators on $H$ and, for an operator $T, G(T)$ denotes its graph. These will be called operator double triangles. A sufficient condition that such a double triangle be non-reflexive is given as Theorem 4. For an operator $T$, $\operatorname{ker} T$ denotes its kernel and $\mathscr{R}(T)$ denotes its range.

\section{Folk theorem}

In finite-dimensional spaces no double triangle is reflexive [5] and none is transitive if the dimension exceeds 2 [4]. The following results seem to be the appropriate generalizations of these facts. The first is known to others.

THEOREM 1. Let $\mathscr{D}_{\mathcal{D}}=\{(0), K, L, M, H\}$ be a double triangle subspace lattice. If any of the vector sums $K+L, K+M, L+M$ is closed, $\mathscr{D}$ is non-reflexive.

Proof. Suppose $K+L$ is closed. Let $Q$ be the projection onto $L$ along $K$, that is, for $x \in H, Q x$ is the unique element of $L$ such that $x-Q x \in K . Q$ is an 
idempotent operator with range $L$ and kernel $K$. Let $\alpha \in \mathbf{C}$ with $\alpha \neq 0,-1$. The spectral mapping theorem shows that $1+\alpha Q$ is invertible. Let $N$ be the subspace $N=(1+\alpha Q) M$. It is easily shown that $N \cap K=N \cap L=N \cap M=(0)$ so $N \notin \mathscr{D}$. However, $N \in \operatorname{Lat} \operatorname{Alg} \mathscr{D}$. For, let $A \in \operatorname{Alg} \mathscr{Q}$. Since $A$ leaves $K$ and $L$ invariant $A Q=Q A$. This, together with the invariance of $M$, gives $A N=$ $A(1+\alpha Q) M=(1+\alpha Q) A M \subseteq(1+\alpha Q) M=N$ and so $N$ is invariant under $A$. The non-reflexivity of $\mathscr{D}$ follows.

If with the above notation neither $\alpha$ nor $\beta$ is 0 or -1 and $\alpha \neq \beta,(1+\alpha Q) M \cap$ $(1+\beta Q) M=(0)$ and it follows that Lat $\operatorname{Alg} \mathcal{D}$ is uncountable. On the other hand $\mathscr{Q}$ is not transitive if $\operatorname{dim} H>2$ as the following shows. For vectors $e, f \in H$ denote by $e \otimes f$ the operator defined by $(e \otimes f) x=(x \mid e) f(x \in H)$ where $(\cdot \mid \cdot)$ denotes the inner-product on $H$.

THEOREM 2. Let $\mathscr{D}=\{(0), K, L, M, H\}$ be a double triangle subspace lattice. If any of the vector sums $K+L, K+M, L+M$ is closed, Alg $\mathscr{D}$ contains an operator of rank two. Consequently Dis is non-transitive if $\operatorname{dim} H>2$.

Proof. Suppose $K+L$ is closed. Let $e \in M$ and $f \in M^{\perp}$ be non-zero vectors. With $Q$ as in the preceding proof put $R=Q^{*} f \otimes Q e-\left(1-Q^{*}\right) f \otimes(1-Q) e . R$ has rank two and, since $R Q=Q R, R$ leaves $K=\operatorname{ker} Q$ and $L=\Re(Q)$ invariant. Let $x \in M$. Then $R x=(Q x \mid f) Q e-((1-Q) x \mid f)(1-Q) e=(Q x \mid f) e \in M$. Thus $R \in \operatorname{Alg} \mathscr{Q}$.

Every double triangle in a two-dimensional space is transitive [3]. No double triangle in any space contains an operator of rank one in its Alg [6].

\section{Operator double triangles}

What can be said concerning the reflexivity of double triangles in which no vector sums, except the trivial ones, are closed. The class of operator double triangles contains many such.

It is easily shown (see [4]) that if $A$ and $B$ are operators on $H$, then $G(A) \cap G(B)=(0)$ if and only if $A-B$ is injective and $G(A) \vee G(B)=H \oplus H$ if and only if $A-B$ has dense range. Also $G(A)+G(B)$ is closed if and only if $A-B$ has closed range. Thus the operators $A, B, C$ give rise to an operator double triangle $\mathscr{D}=\{(0), G(A), G(B), G(C), H \oplus H\}$ if and only if each of $A-B, A-C, B-C$ is injective with dense range. The 'no closed vector sum' case occurs precisely when none of these three difference operators is invertible. 
In general, two double triangles $\mathscr{D}_{i}=\left\{(0), K_{i}, L_{i}, M_{i}, H_{i}\right\}(i=1,2)$ are said to be similar if there is a bounded bijection $S: H_{1} \rightarrow H_{2}$ such that $S K_{1}=K_{2}$, $S L_{1}=L_{2}, S M_{1}=M_{2}$. Reflexivity and (transitivity) is a similarity invariant since $S$ Lat Alg $\mathscr{D}_{1}=$ Lat Alg $\mathscr{D}_{2}$ when $\mathscr{D}_{1}$ and $\mathscr{D}_{2}$ are similar (by $S$ ).

The above remark reduces the study of operator double triangles and their reflexivity (and their transitivity) to the study of those of the form $\mathscr{D}=$ $\{(0), G(A), G(B), G(0), H \oplus H\}$ since $\{(0), G(A), G(B), G(C), H \oplus H\}$ is similar to $\{(0), G(A-C), G(B-C), G(0), H \oplus H\}$ by $S=\left[\begin{array}{cc}1 & 0 \\ -C & 1\end{array}\right]$. If the set of operators on $H \oplus H$ is identified in the usual way with the set of $2 \times 2$ matrices whose entries are operators on $H$, the Alg of the double triangle $\{(0), G(A), G(B), G(0), H \oplus H\}$ consists precisely of those operators of the form $\left[\begin{array}{cc}X & Z \\ 0 & Y\end{array}\right]$ for which $Y A=A X+A Z A$ and $Y B=B X+B Z B$.

THEOREM 3. Let $D=\{(0), G(A), G(B), G(0), H \oplus H\}$ be an operator double triangle. If there exists a complex scalar $\lambda$ such that

(i) $\Re(A)+\Re(B) \subseteq \Re(A-\lambda B)$ and

(ii) the image of $\operatorname{ker}(A-\lambda B)$ under $A$ is not dense in $H$, then $\mathscr{D}$ is non-reflexive.

Proof. Suppose $\lambda$ is such a scalar. Let $P$ be the orthogonal projection with range $\operatorname{ker}(A-\lambda B)^{\perp}$. Then $\mathcal{R}(A(1-P))$ is not dense in $H$. Since $(A-\lambda B)$ $(1-P)=0, A(1-P)=\lambda B(1-P)$. Since $\mathcal{R}(A) \subseteq \Re(A-\lambda B)$ and $R(B) \subseteq$ $\Re(A-\lambda B), A=(A-\lambda B) F$ and $B=(A-\lambda B) E$ for some operators $E$ and $F$ on $H$ [1]. These operators may be taken to satisfy $P E=E$ and $P F=F$. Since $B-A$ is injective and $B-A=(A-\lambda B)(E-F), E-F$ is injective. Also, $A-\lambda B=(A-\lambda B)(F-\lambda E)=(A-\lambda B) P$ so $F-\lambda E=P$ since $A-\lambda B$ is injective on $\Re(P)$.

Since $B=(A-\lambda B) E, A E=B(1+\lambda E)$. Let $C=A E=B(1+\lambda E)$. Clearly $\Re(C) \subseteq \Re(A) \cap \mathcal{R}(B)$ and $\Re(A(1-P)) \subseteq \Re(A) \cap \Re(B)$. Thus $\Re(C)+$ $\Re(A(1-P)) \subseteq \Re(A) \cap \Re(B)$. The reverse inclusion is also true. For, let $x \in$ $\Re(A) \cap \Re(B)$. Then $x=A y=B z$ for some vectors $y, z \in H$ and $x=$ $(A-\lambda B) F y=(A-\lambda B) E z$ gives $F y=E z$. Hence $F y=P y+\lambda E y=E z$ so $P y$ $=E(z-\lambda y)$. Thus $y=E(z-\lambda y)+(1-P) y$ and $x=A y=C(z-\lambda y)+$ $A(1-P) y \in \Re(C)+\Re(A(1-P))$. So $\Re(A) \cap \Re(B)=\Re(C)+$ $\Re(A(1-P))$.

Let $\left[\begin{array}{ll}X & Z \\ 0 & Y\end{array}\right] \in$ Alg $\mathcal{D}$. Then $Y A=A X+A Z A$ and $Y B=B X+B Z B$. The first of these equations yields

$$
\begin{gathered}
Y C=A X E+A Z C, \\
Y A(1-P)=A X(1-P)+A Z A(1-P),
\end{gathered}
$$


and the second gives

$$
\begin{aligned}
Y C & =B X(1+\lambda E)+B Z C, \\
Y A(1-P) & =\lambda B X(1-P)+B Z A(1-P) .
\end{aligned}
$$

Since $Y$ leaves the linear manifolds $\mathscr{R}(A)$ and $\mathscr{R}(B)$ invariant, it leaves $\Re(C)+$ $\Re(A(1-P))$ invariant. Let $x, y \in H$. Then $Y C x+Y A(1-P) y=C x^{\prime}+$ $A(1-P) y^{\prime}$ for some vectors $x^{\prime}$ and $y^{\prime}$. We now find $x^{\prime}$ and $(1-P) y^{\prime}$ in terms of $x$ and $y$. From (1) and (2),

$$
Y C x+Y A(1-P) y=A[X E x+Z C x+X(1-P) y+Z A(1-P) y] \text {. }
$$

Since this also equals $A\left[E x^{\prime}+(1-P) y^{\prime}\right]$, it follows that

$$
X E x+Z C x+X(1-P) y+Z A(1-P) y=E x^{\prime}+(1-P) y^{\prime} .
$$

From (3) and (4),

$$
\begin{aligned}
Y C x+Y A(1-P) y \\
=B[X(1+\lambda E) x+Z C x+\lambda X(1-P) y+Z A(1-P) y] .
\end{aligned}
$$

Since this also equals $B\left[(1+\lambda E) x^{\prime}+\lambda(1-P) y^{\prime}\right]$, it follows that

$$
\begin{aligned}
X(1+\lambda E) x+Z C x+\lambda X(1-P) y+Z A & (1-P) y \\
& =(1+\lambda E) x^{\prime}+\lambda(1-P) y^{\prime}
\end{aligned}
$$

Subtracting $\lambda$ times (5) from 6 gives

$$
X x+(1-\lambda) Z C x+(1-\lambda) Z A(1-P) y=x^{\prime} .
$$

Substituting this into (5) gives

$$
\begin{aligned}
X E x-E X x+(1 & -(1-\lambda) E) Z C x+X(1-P) y \\
& +(1-(1-\lambda) E) Z A(1-P) y=(1-P) y^{\prime}
\end{aligned}
$$

Since for all vectors $x, y \in H, Y C x+Y A(1-P) y=C x^{\prime}+A(1-P) y^{\prime}$ where $x^{\prime}$ and $(1-P) y^{\prime}$ are given by the last two equations, it follows that

$$
\text { (7) } \begin{aligned}
Y C= & C[X+(1-\lambda) Z C] \\
& +A(1-P)[X E-E X+(1-(1-\lambda) E) Z C], \\
\text { (8) } Y A(1-P)= & C[(1-\lambda) Z A(1-P)] \\
& +A(1-P)[X(1-P)+(1-(1-\lambda) E) Z A(1-P)]
\end{aligned}
$$

for every element $\left[\begin{array}{ll}X & Z \\ 0 & Y\end{array}\right]$ of $\mathrm{Alg} \mathscr{Q}$.

The proof that $\mathscr{D}$ is non-reflexive is completed by considering two cases.

First, suppose $\lambda \neq 0,1$. Define the subspace $M$ of $H \oplus H$ by $M=$ $G((1-\lambda) C) \vee\{(0) \oplus \overline{\mathscr{R}(A(1-P))}\}$ where ${ }^{\text {" }}$, denotes norm closure. Let [ $\left.\begin{array}{ll}X & Z \\ 0 & Y\end{array}\right]$ $\in \operatorname{Alg} \mathscr{D}$ and let $x, y \in H$. Then

$$
\left[\begin{array}{ll}
X & Z \\
0 & Y
\end{array}\right]\left[\begin{array}{c}
x \\
(1-\lambda) C x+A(1-P) y
\end{array}\right]=\left[\begin{array}{c}
X x+(1-\lambda) Z C x+Z A(1-P) y \\
(1-\lambda) Y C x+Y A(1-P) y
\end{array}\right]
$$


and, using (7) and (8), this equals

$$
\left[\begin{array}{c}
v \\
(1-\lambda) C v+A(1-P) w
\end{array}\right]
$$

with $v=X x+(1-\lambda) Z C x+Z A(1-P) y$ and $w=(1-\lambda) X E x-(1-\lambda)$ $\cdot E X x+(1-\lambda)(1-(1-\lambda) E) Z C x+X(1-P) y+(1-(1-\lambda) E) Z A$ $\cdot(1-P) y$. From this it follows that $M \in$ Lat Alg $\mathscr{Q}$.

Clearly $M \neq(0)$. If $z \in \overline{\mathscr{R}(A(1-P))^{\perp}}$, then $-(1-\bar{\lambda}) C^{*} z \oplus z \in M^{\perp}$ so $M \neq$ $H \oplus H$. $C$ is injective, since $C u=0$ implies $E u=0=u+\lambda E u$, so $M \neq G(0)$. $M=G(A)$ or $G(B)$ implies $P=1$ and $(1-\lambda) C=A$ or $B$. From this would follow $E=F$ which contradicts the injectivity of $E-F$. Thus $M \neq G(A)$ or $G(B)$. So $M \notin \mathscr{D}$ and $\mathscr{D}$ is non-reflexive.

Finally, suppose $\lambda=0$ or 1 . Then $P=1, F=1+\lambda E$ and $\Re(A) \cap \mathscr{R}(B)=$ $\Re(C)$. Let $\mu$ be a scalar, neither 0 nor 1 , such that $F-\mu E$ is invertible. Such a $\mu$ exists. For, if $\lambda=0$, choose $\mu=1 / \gamma$ where $\gamma \neq 0,1$ and $E-\gamma$ is invertible and, if $\lambda=1$, choose $\mu=1+1 / \rho$ where $\rho \neq 0,-1$ and $E-\rho$ is invertible. Define the subspace $N$ of $H \oplus H$ by $N=G(D)$ where $D=(1-\mu) C(F-\mu E)^{-1}$. Let $\left[\begin{array}{ll}X & Z \\ 0 & Y\end{array}\right]$ $\in A \operatorname{Alg} \mathscr{D}$ and let $x \in H$. There exists $x^{\prime} \in H$ such that $Y C x=C x^{\prime}$. Equations (5) and (6) give $X E x+Z C x=E x^{\prime}$ and $X F x+Z C x=F x^{\prime}$. Subtracting $\mu$ times the former from the latter gives $x^{\prime}=(F-\mu E)^{-1}[X(F-\mu E)+(1-\mu) Z C] x$. Thus

$$
Y C=C(F-\mu E)^{-1} X(F-\mu E)+(1-\mu) C(F-\mu E)^{-1} Z C .
$$

Hence

$$
\begin{aligned}
Y D-D X & =(1-\mu) Y C(F-\mu E)^{-1}-(1-\mu) C(F-\mu E)^{-1} X \\
& =(1-\mu)\left[Y C-C(F-\mu E)^{-1} X(F-\mu E)\right](F-\mu E)^{-1} \\
& =(1-\mu)\left[(1-\mu) C(F-\mu E)^{-1} Z C\right](F-\mu E)^{-1} \quad(\operatorname{using}(9)) \\
& =\left[(1-\mu) C(F-\mu E)^{-1}\right] Z\left[(1-\mu) C(F-\mu E)^{-1}\right] \\
& =D Z D,
\end{aligned}
$$

so $G(D)$ is invariant under $\left[\begin{array}{ll}X & Z \\ 0 & Y\end{array}\right]$. Thus $N \in \operatorname{Lat} A \operatorname{Alg} \mathscr{Q}$. It is easily shown that $N \notin \mathscr{D}$ so $\mathscr{D}$ is non-reflexive.

This completes the proof of the theorem.

If $\lambda=0$ or 1 in the above theorem, Lat $A \lg \mathscr{D}$ is uncountable since the set $\{\mu \in \mathrm{C}: \mu \neq 0,1$ and $F-\mu E$ is invertible $\}$ is uncountable and each of its elements $\mu$ gives a different element $G\left((1-\mu) C(F-\mu E)^{-1}\right)$ of Lat Alg $\mathscr{Q}$.

If $\{(0), G(A), G(B), G(0), H \oplus H\}$ is an operator double triangle in which $\Re(A) \cap \Re(B)$ is neither $(0)$ nor dense, it is non-reflexive since $H \oplus L \in$

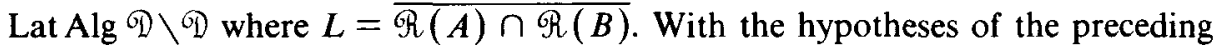


theorem, $\Re(A) \cap \Re(B)=\mathscr{R}(C)+\mathscr{R}(A(1-P))$ cannot be $(0)(C$ is injective $)$ but may be dense, for example, if $\lambda=0$.

Corollary 1. Let $\mathscr{D}=\{(0), G(A), G(B), G(0), H \oplus H\}$ be an operator double triangle. If there exists a complex scalar $\lambda$ such that either

(i) $\Re(A)+\Re(B) \subseteq \Re(A-\lambda B)$ and

(ii) $A-\lambda B$ is injective, or

(iii) $A-\lambda B$ is invertible,

then 10 is non-reflexive.

Consideration of the case $\lambda=0$ leads to the following.

Corollary 2. Let $\mathscr{Q}=\{(0), G(A), G(B), G(0), H \oplus H\}$ be an operator double triangle. If $\Re(A)$ and $\Re(B)$ are comparable, $\mathscr{Q}$ is non-reflexive.

Note that, even if $\{(0), G(A), G(B), G(0), H \oplus H\}$ is an operator double triangle, the condition $\mathcal{R}(A)+\mathscr{R}(B) \subseteq \Re(A-\lambda B)$ need not imply nor be implied by the condition $A-\lambda B$ is injective. (Consider $A=S^{*}-1, B=1$, where $S$ is the unilateral shift of multiplicity 1 , and $\lambda=-1, \lambda=0$.) Also, these two conditions together need not imply that $A-\lambda B$ is invertible since, for example, both $A$ and $B$ may be compact (on an infinite-dimensional space) with $\Re(A)$ and $R(B)$ comparable.

By recalling that the operator double triangles

$$
\{(0), G(A), G(B), G(C), H \oplus H\}
$$

and

$$
\{(0), G(A-C), G(B-C), G(0), H \oplus H\}
$$

are similar we obtain slightly more general results exemplified by the following.

TheOREM 4. Let $\mathscr{D}=\{(0), G(A), G(B), G(C), H \oplus H\}$ be an operator double triangle. If there exist complex scalars $\alpha, \beta$ such that

(i) $\Re(A-C)+\Re(B-C) \subseteq \Re(\alpha(A-C)+\beta(B-C))$ and

(ii) the image of $\operatorname{ker}(\alpha(A-C)+\beta(B-C))$ under $A-C$ is not dense in $H$, then $\mathscr{D}$ is non-reflexive.

RemarKs 1. The question: Is every operator double triangle in Hilbert-space non-reflexive? remains unanswered.

2. There might be a double triangle subspace lattice that is not similar to any operator double triangle but none have yet been exhibited. 
3. The existence of a transitive operator double triangle has yet to be established. It seems likely that no operator double triangle satisfying the hypotheses of Theorem 3 is transitive, particularly since the transitive candidate discussed in Theorem 2 of [2] fails to satisfy them (because $\Re(A) \cap \Re(B)=(0)$ ).

\section{References}

[1] R. G. Douglas, 'On majorization, factorization and range inclusion of operators in Hilbert space,' Proc. Amer. Math. Soc. 17 (1966), 413-416.

[2] D. W. Hadwin, W. E. Longstaff and Peter Rosenthal, 'Small transitive lattices,' Proc. Amer. Math. Soc. 87 (1983), 121-124.

[3] P. R. Halmos, 'Finite-dimensional Hilbert spaces,' Amer. Math. Monthly 77 (1970), 457-464.

[4] P. R. Halmos, 'Ten problems in Hilbert space,' Bull. Amer. Math. Soc. 76 (1970), 887-933.

[5] P. R. Halmos, 'Reflexive lattices of subspaces,' J. London Math. Soc. 4 (1971), 257-263.

[6] W. E. Longstaff, 'Strongly reflexive lattices,' J. London Math. Soc. 11 (1975), 491-498.

[7] W. E. Longstaff and Peter Rosenthal, 'On two questions of Halmos concerning subspace lattices,' Proc. Amer. Math. Soc. 75 (1979), 85-86.

[8] Gábor Szász, Introduction to lattice theory, 3rd ed. (Academic Press, New York and London, 1963).

Department of Mathematics

University of Western Australia

Nedlands, Western Australia 6009 